\title{
Toward perfect reads: short reads correction via mapping on compacted de Bruijn graphs
}

\author{
Antoine Limasset ${ }^{1,}$, Jean-François Flot ${ }^{1, *}$, and Pierre Peterlongo ${ }^{2, *}$ \\ ${ }^{1}$ Université libre de Bruxelles, Evolutionary Biology \& Ecology, C.P. 160/12, Avenue F.D. Roosevelt 50, 1050 Bruxelles, Belgium \\ ${ }^{2}$ Univ Rennes, Inria, CNRS, IRISA, F-35000 Rennes, France \\ *Co-last authors with equal contributions. \\ Associate Editor: D
}

Received on D; revised on D; accepted on D

\begin{abstract}
Motivations Short-read accuracy is important for downstream analyses such as genome assembly and hybrid long-read correction. Despite much work on short-read correction, present-day correctors either do not scale well on large data sets or consider reads as mere suites of k-mers, without taking into account their full-length read information.

Results We propose a new method to correct short reads using de Bruijn graphs, and implement it as a tool called Bcool. As a first step, Bcool constructs a compacted de Bruijn graph from the reads. This graph is filtered on the basis of $k$-mer abundance then of unitig abundance, thereby removing most sequencing errors. The cleaned graph is then used as a reference on which the reads are mapped to correct them. We show that this approach yields more accurate reads than $k$-mer-spectrum correctors while being scalable to human-size genomic datasets and beyond.

Availability and Implementation The implementation is open source and available at http: //github.com/Malfoy/BCOOL under the Affero GPL license and as a Bioconda package.

Contact Antoine Limasset anto ine. limasset @gmail. com \& Jean-François Flot jflot@ulb. ac . be

\& Pierre Peterlongo pierre.peterlongo@inria.fr
\end{abstract}

\section{Introduction}

1.1 Why correct reads?

Genome sequencing is a fast-changing field. Two decades have seen three generations of sequencing technologies: Sanger electropherograms (a.k.a. first-generation sequencing), short reads from second-generation sequencing (SGS) and long, error-prone reads from third-generation sequencing (TGS). Albeit powerful, these technologies all come with stochastic errors, and for some, non-stochastic ones. Stochastic errors are usually corrected using a consensus approach leveraging the high coverage depth available in most genomic projects, whereas non-stochastic errors can be eliminated by "polishing" the sequences using reads obtained from a different sequencing technique.

In de novo assemblers following the overlap-layout-consensus (OLC) paradigm, the stochastic errors present in the reads are handled during the consensus step toward the end of the process. By contrast, tools following the de Bruijn graph (DBG) paradigm attempt to filter out erroneous $k$-mers by considering only $k$-mers present at least a minimal number of times in the reads to be assembled. Both paradigms may benefit from a preliminary error correction step. In the case of DBG assemblers, lowering the error rate in the reads to be assembled makes it possible to use a greater $k$-mer size, paving the way for a more contiguous assembly. In OLC assemblers it allows more stringent alignment parameters to be used, thereby improving the speed of the process and reducing the amount of spurious overlaps detected between reads.

Beyond de novo assembly, other applications that require mapping, such as SNP calling, genotyping or taxonomic assignation, may also benefit from a preliminary error-correction step aimed at increasing the signal/noise ratio and/or reducing the computational cost of detecting errors a posteriori [1,2].

\subsection{On the use of short reads as long reads are rising}

Although long-read technologies from third-generation sequencing (TGS), marketed by Pacific Biosciences (PacBio) and Oxford Nanopore Techonologies (ONT), are on the rise and may surpass short reads for many purposes such as genome assembly (as TGS reads are order of magnitude longer and are therefore less sensible to repeats [3]), we have reasons to think that SGS will still be broadly used in the next decade. This is because SGS reads remain considerably cheaper, and their accurate sequences make them highly valuable. Besides, recent 
methods are able to deliver long-distance information based on short-read sequencing. Chromosome conformation capture [4] provides pairs of reads that have a high probability to originate from the same chromosome and Chromium 10X [5] uses a droplet mechanism to ensure that a pack of reads come from a single DNA fragment of up to hundreds of thousands base pairs. Both of these techniques have been shown to produce assembly continuity comparable to TGS assembly $[6,7,8]$. SGS reads are also used jointly with long reads to compensate the latter's high error rate (and systematic homopolymer errors in the case of ONT reads [9]) in a costefficient way [10]. These different applications make it worth investing effort in improving short-read correctors beyond the current state of the art, in the hope that near-perfect reads will positively impact all downstream analyses that require accurate sequences.

\section{$1.3 k$-mer-spectrum techniques}

$k$-mer-spectrum techniques are conceptually the simplest correction method and remain broadly used. The underlying intuition is that true genomic $k$-mers will be seen many times in the read set, whereas erroneous $k$-mers originating from sequencing errors will be comparatively much rarer. The first step to correct reads using this approach is therefore to choose an abundance threshold (above which $k$-mers are called "solid" and below which they are called "weak" [11]). $k$-mer-spectrum correctors aim to detect all weak $k$-mers in the reads and correct them by turning them into solid ones.

One of the best $k$-mer-spectrum correctors available to date [12] is Musket [13]; however, its memory consumption is high on large genomes because of its indexing structure. Another tool, Bloocoo [14], achieves a comparatively lower memory footprint, even on genomes comprising billions of bases (such as the human one), by using a Bloom filter to index $k$ mers. Lighter [15] also uses Bloom filters but bypasses the $k$-mer counting phase by only looking at a subset of the $k$-mers in a given data set, therefore achieving greater speed. One problem with these tools is that they rely on local $k$-mer composition with a bounded $k$-mer size $(<=32)$, which limits correction power on complex data sets. A less "local" approach is implemented in the BFC [16] corrector, which attempts to correct each read as a whole by finding the minimal number of substitutions required for a read to be entirely covered by solid $k$-mers.

\subsection{Other read correction techniques}

Other correction techniques rely either on suffix arrays (allowing the use of substrings of various sizes instead of only fixed $k$-length words) [17] or on multiple-read alignments [18]. Despite their methodological appeal, these techniques are resource-expensive and do not scale well on large data sets. Moreover, benchmarks suggest that they perform significantly worse than state-of-the-art $k$-mer-spectrum correctors [19]. Yet another approach for correcting reads, pioneered by LoRDEC [20] then by LoRMA [21], is to use de Bruijn graphs instead of strings as a basis for correction. In the LoRDEC approach, this de Bruijn graph is built from highly accurate short reads and used to correct long reads. In LoRMA, the de Bruijn graph is built from the very same long reads that the program is attempting to correct. Both programs were engineered to handle long reads and it is surprising that such DBG-based approach was never applied till now to correct highly accurate SGS short reads such as those produced by Illumina sequencers. Finally, Rcorrector [22] relies on an abundance-annotated de Bruijn graph to correct Illumina RNA-seq reads. Despite its interest, this approach hardly scales to large genomic datasets and yields lower result quality compared to the tools tailored for genomic datasets.

Here, we implement a DBG-based corrector geared towards Illumina reads, which are characterized by errors that are solely substitutions an affect less than one percent of the output bases. We then show that this corrector, dubbed Bcool, vastly outperforms state-of-the-art $k$-merspectrum correctors while being both scalable and resource-efficient.

\section{Method}

The intuition behind $k$-mer-spectrum correction is that $k$-mers, once filtered according to their abundance, represent a reference that can be used to correct the reads. The idea that a de Bruijn graph provides a better reference than a $k$-mer set might be surprising at first glance since a de Bruijn graph is equivalent to its set of $k$-mers.

However, a novelty of our approach is that we build a compacted DBG, that is, a DBG in which non-branching paths are turned into unitigs. We are therefore able to remove erroneous $k$-mer relying on the graph topology rather than on their abundance alone. This results in a better distinction between erroneous and genomic $k$-mers. The second novelty is to rely on whole read alignments on this cleaned graph, performing a global correction of a read and allowing the use of high $k$-mer size for an improved correction on repetitive or large genomes.

After briefly reviewing several limitations of $k$-mer-spectrum correction (Section 2.1), we detail how our proposed approach manages to tackle these issues while describing some key parts of our workflow (Section 2.2)

\section{$2.1 k$-mer spectrum limitations}

In this section we identify four sources of miscorrection in $k$-mer spectrum approaches, as represented in Figure 1. As mentioned previously, $k$-merspectrum correctors infer a set of solid $k$-mers that are used for correcting reads. Erroneous $k$-mers (i.e., $k$-mers containing at least one sequencing error) can be filtered out by keeping only $k$-mers above a given abundance threshold called the solidity threshold. $k$-mers whose abundance is higher or equal (respectively lower) to this threshold are called solid (respectively weak).

(1) Weak genomic k-mers. Depending on the solidity threshold chosen, random variations in sequencing depth may cause some genomic $k$-mers to fall below the threshold and be erroneously filtered out. This kind of $k$-mer creates a situation such as the one represented in Figure 1.1, where an isolated weak $k$-mer is flagged as a putative error on a read that is actually correct. Since at least $k$ successive weak $k$-mers are expected to be seen when there is a sequencing error (Figure 2), $k$-mer spectrum-based correctors normally consider isolated weak $k$-mers as likely to be simply missed genomic $k$-mers and do not attempt to correct them.

(2) Solid erroneous $k$-mers. Conversely, setting the solidity threshold too low may lead to the inclusion of some erroneous $k$-mers in the trusted set of solid $k$-mers (Figure 1.2). As a result, the errors in the reads harboring such $k$-mers are not corrected and may also propagate to other reads if these $k$-mers are used for correction.

(3) Errors covered by solid k-mers If a sequencing error in a read is covered partly or entirely by genomic $k$-mers originating from other regions of the genome, the error may not be detected and it may be difficult to correct as the remaining isolated weak $k$-mers will be hard to distinguish from the pattern in point 1 above. Such situations are likely to occur in repeated or quasi-repeated genome regions, leading to complex situations where genomic sequences may have several contexts.

(4) Nearby errors Accurate correction is also complex when multiple errors occur nearby each other (i.e., less than $k$ bases apart). In such situations, the number of errors and their positions cannot be easily estimated, and $k$-mer-spectrum correctors have to perform a very large number of queries to correct them. Musket uses an aggressive greedy 


\begin{tabular}{|c|c|c|c|c|}
\hline & 1) Weak genomic $k$-mers & 2) Solid erroneous $k$-mers & 3) Errors covered by solid $k$-mers & 4) Nearby errors \\
\hline reference & GCTGATCGCTAGTT & GCTGATCGCTAGTT & GCTGATCGCTAGTT....AGCTCTTT & GCTGATCGCTAGTT \\
\hline $\begin{array}{l}\text { set of solid } \\
k \text {-mers } \\
\text { erroneous bases }\end{array}$ & 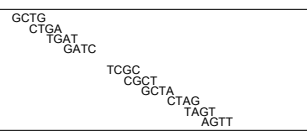 & 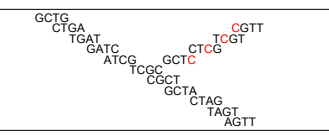 & 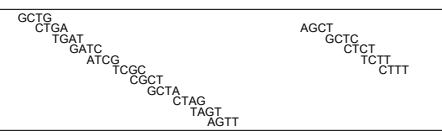 & 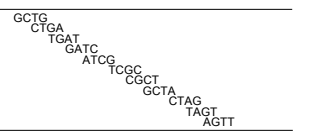 \\
\hline \multirow{2}{*}{$\begin{array}{l}\text { example } \\
\text { of read } \\
\text { weak } k \text {-mers } \\
\text { solid } k \text {-mers }\end{array}$} & GCTGATCGCTAGTT & GCTGATCGCTCGTT & GCTGATCGCTCGTT & GCTGAGCCCTAGTT \\
\hline & $\begin{array}{l}\text { ATCG is genomic } \\
\text { but weak }\end{array}$ & $\begin{array}{c}\text { GCTC is erroneous } \\
\text { but solid }\end{array}$ & $\begin{array}{l}\text { GCTC is solid as it exists } \\
\text { somewhere else in the genome }\end{array}$ & $\begin{array}{l}\text { two nearby errors in read } \\
\text { result in six weak } k \text {-mers }\end{array}$ \\
\hline
\end{tabular}

\begin{tabular}{|c|c|c|c|c|}
\hline & 1) Weak genomic $k$-mers & 2) Solid erroneous $k$-mers & 3) Errors covered by solid $k$-mers & 4) Nearby errors \\
\hline reference & GCTGATCGCTAGTT & GCTGATCGCTAGTT & GCTGATCGCTAGTT...AGCTCTTT & GCTGATCGCTAGTT \\
\hline $\begin{array}{l}\text { example } \\
\text { of read } \\
\text { erroneous bases }\end{array}$ & GCTGATCGCTAGTT & GCTGATCGCTCGTT & GCTGATCGCTCGTT & GCTGAGCCCTAGTT \\
\hline $\begin{array}{l}\text { Bcool } \\
\text { solution } \\
\text { - mismatches }\end{array}$ & $\overline{\text { GCTGATCGCTAGTT }}$ & $\begin{array}{c}\text { C†CGTT } \\
\text { GCTGATCGCTA } \\
\text { CTAGTTT }\end{array}$ & $\begin{array}{l}\text { GCTÅTTT } \\
\text { GCTGATCGCT } \\
\text { GCTCTIT mapping } \\
\text {...AGCT }\end{array}$ & $\overline{\text { GCTGATCGCTAGTT }}$ \\
\hline
\end{tabular}

Fig. 1. Top: Four issues with $k$-mer-spectrum methods. 1) Genomic $k$-mers may be appear weak because of their low abundance. 2) Erroneous $k$-mers may appear solid because of their high abundance. 3) Sequencing errors may be validated by genomic $k$-mers originating from other parts of the genome. 4) Multiple errors may occur on a $k$-mer, resulting in a large weak region.

Bottom: How Bcool handles the problems highlighted (Blue half-arrows represent the paths of the graph on which given read maps). 1) By using a very low $k$-mer abundance threshold coupled with a unitig abundance threshold, Bcool retains low-abundance $k$-mers and manages to correct the reads that contain them. 2) Bcool detects the tip pattern produced by solid erroneous $k$-mers and is therefore able to discard them. Other erroneous $k$-mers are detected at the unitig filtering step. 3) By considering mappings globally, Bcool chooses the best path for each read, i.e., the one on which it maps with the smallest number of mismatches. 4) Bcool uses unitigs instead of $k$-mers to correct reads and is therefore able to deal with reads that contain several nearby errors.

\section{GCTGATC CTACGTTCATGC}

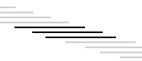

GCTGATC $C$ TACGTTCATGC

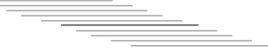

Fig. 2. Patterns of weak $k$-mers resulting from a sequencing error, depending on $k$. Sequencing errors in the read are pictured in grey. Solid and weak $k$-mers are represented respectively with black and grey lines, with $k=4$ (left) and $k=8$ (right). The $k$-mer pictured in darker grey contain two errors. A sequencing error usually impacts $k k$-mers and creates a weak region of size $2 * k-1$ (left). Choosing a large $k$ results in most $k$-mers of a read being weak as they contain one or several errors (right).

heuristic that tries to replace the first weak $k$-mer encountered by a solid one then checks whether the next $k$-mers became solid as a result. But, as shown in Figure 1.4, this heuristic is inefficient if the $k$-mers that follow contain other sequencing errors.

$k$-mer size All the issues highlighted above boil down to a central problem when using $k$-mer-spectrum correctors: the size of $k$. If a too large $k$ mer size is used, most $k$-mers contain at least one sequencing error and many of them actually contain several errors (Figure 2). In those cases $k$-mer-spectrum correctors may be unable to locate errors and to perform correction as they rely on genomic $k$-mers to find possible candidates to replace weak ones. On the contrary, if $k$ is too small, most $k$-mers are solid and almost no correction is performed. As $k$-mer-spectrum correctors are usually geared towards correcting SGS reads, they use a $k$-mer size around 31 that is well suited for the error rate of Illumina data. Choosing a larger $k$ results in sub-optimal correction or even in a failure of the program to run

(see Supplementary materials). This limitation may be a problem when addressing large and repeat-rich genomes, as a large number of $k$-mers are repeated in various contexts throughout the genomes and large $k$-mers are required in order to distinguish them.

\subsection{DBG-based reads correction}

In this section we describe our proposal, called Bcool (which stands for "de Bruijn graph-based read correction by alignment"). The basic idea is to construct a DBG from the read set, to clean it, and then to map the reads on the DBG. Reads that map with less than a threshold number of mismatches are corrected using the graph sequence, which is supposed to be almost error-free. An important Bcool feature is that the graph is constructed by filtering out low-coverage $k$-mers and additionally by discarding unitigs according to topological information (see Section 2.2.2), yielding a almost perfect reference graph.

We present in Figure 1 some simple examples illustrating how our DBG-based read correction handles the problems highlighted in Figure 1. Our proposal differs from $k$-mer-spectrum techniques in that our reference is a cleaned compacted DBG [23] instead of a set of $k$-mers, and that we map the reads onto the graph instead of looking at all $k$-mers contained in the reads. With highly covered data, our approach also allows the use of large $k$-mer size, improving correction of complex regions.

Bcool's workflow is depicted in Figure 3. Each of its components is either an independent tool already published or an independent module that could be reused in other frameworks. We describe below the different key steps of the workflow. 


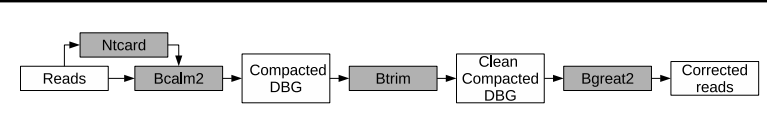

Fig. 3. Bcool workflow. The white boxes are FASTA files and the grey boxes represent the tools that process or generate them. Ntcard [24] is used to select the best-suited $k$-mer size. A compacted DBG is then constructed using Bcalm2 [25]. The Btrim [26] module cleans the graph, and the reads are finally mapped back on the de Bruijn graph using Bgreat2 [27].

\subsubsection{DBG construction}

In Bcool, the DBG is constructed using Bcalm2 [25], a resource-efficient method to build a compacted DBG. In a compacted DBG, nodes are not composed of single $k$-mers but of unitigs (i.e., maximal simple paths of the DBG) of lengths larger or equal to $k$. As explained in more detail in the next section, Bcool uses a seed-and-extend mapping strategy with seeds of lengths less than or equal to the $k$ parameter used for graph construction. This allows Bcool to correct reads that do not contain any solid $k$-mer, as long as these reads contain at least one error-free seed and align on the graph. Thus, Bcool can use a large $k$-mer size and is therefore less affected by genomic repeats than $k$-mer-spectrum correctors. Moreover, with a large $k$ value, most sequencing errors generate a tip in the graph rather than a bubble. Bcool takes advantage of this by performing a graph-correction step aimed at removing tips.

\subsubsection{DBG clean-up}

A novelty of this work, made possible thanks to the compacted graph representation, is the way we clean up the reference DBG before using it to correct reads. In this context we propose and use the notion of unitig abundance, defined as the the mean abundance of all its constituent $k$-mers

The DBG is initially constructed with a very low abundance threshold (by default 2, i.e. $k$-mers that occur only once are considered as probable errors and discarded). This very low threshold value decreases the probability of missing genomic $k$-mers, but as a consequence, many erroneous $k$-mers are not filtered out. A first step to remove those is to remove short dead-ends (a.k.a 'tips'). Formally, we define a tip as a unitig of length inferior to $2 *(k-1)$ that has no successor at one of its extremities. Such dead-ends mainly result from sequencing errors occurring on the first or last $k$ nucleotides of a read. By contrast, errors located at least $k$ nucleotides away from the start or the end of a read form bubble-like patterns, and such errors are detected and filtered based on unitig coverage. In this second step, we tackle remaining erroneous $k$ mers by taking a look at unitig abundance. We choose a unitig abundance threshold (higher than the $k$-mer abundance threshold used previously) and when an unitig has an abundance lower than this threshold, we discard it completely. Intuitively, averaging the abundance across each unitig makes it possible to 'rescue' genomic $k$-mers with low abundance (that tend to be lost by $k$-mer-spectrum techniques, see Figure 1.1) by detecting that they belong to high-abundance unitigs, and these genomic $k$-mers can then be used for correction. On the other hand, erroneous $k$-mers are likely to belong to low-abundance unitigs that are filtered out. The unitig threshold can be user-specified or can be inferred by looking at the unitig abundance distribution and choosing the first local minimum. These cleaning steps are applied several time in an iterative manner for handling complex scenarios where error patterns are nested. Together, those two approaches allow us to address the issues depicted in Figure 1.1 and Figure 1.2. Compared with a strategy based only on $k$-mer abundance, our approach keeps more lowabundance genomic $k$-mers while removing more erroneous $k$-mers. As shown in the Supplementary materials using simulated data, the filtering strategy used by Bcool produces a graph with less erroneous $k$-mers (False Positive $k$-mers) and less missed genomic $k$-mers (False Negative) $k$ mers than the sole $k$-mer-abundance threshold used by $k$-mer-spectrum correctors, resulting in a better set of $k$-mers. Detailed evaluation of the tipping and unitig-filtering strategies is provided in the Supplementary Materials.

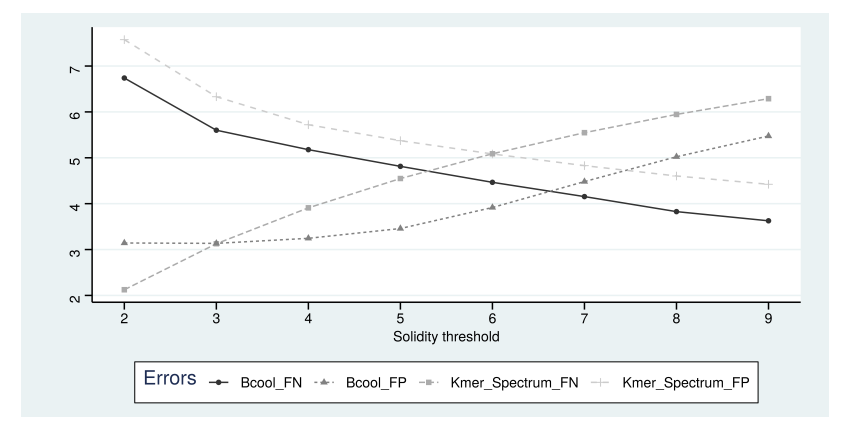

Fig. 4. Impact of the solidity threshold on the $k$-mer selection according to the strategy used. The $y$-axe shows the number of errors, that are either false positives $k$-mers (FP, erroneous $k$-mers that are retained in the $k$-mer set) or false negatives $k$-mers (FN, genomic $k$-mers that are discarded). In this experiment we used $k=63$ on a 50X coverage of 150-bp reads simulated from the C. elegans reference genome. For $k$-mer-spectrum techniques, the solidity threshold applies to $k$-mers, while for Bcool it applies to the unitigs constructed from non-unique $k$-mers. We observe that the proposed graph cleaning operations are able to retain more genomic $k$-mers and remove more erroneous $k$-mers than the raw $k$-mers abundance threshold.

\subsubsection{Read mapping}

In contrast to $k$-mer spectrum-based techniques, Bcool uses an explicit representation of the DBG. Although in its current implementation this entails a higher memory usage and computational cost than $k$-merspectrum correctors, doing so provides an efficient way to fix the issues depicted in Figure 1.3 and Figure 1.4. Each read is aligned in full length on the graph, and the correction is made on the basis of the most parsimonious path on which the read maps in the graph. For mapping reads on the DBG, we use an improved, yet unpublished, version of Bgreat [28] called "Bgreat2". The description of this method is out of the scope of this paper but we provide here the main characteristics of the new implementation. The main improvements are that Bgreat2 has no thirdparty dependencies (in contrary to the published version) and that it outputs optimal alignments among the candidates. The alignment procedure uses a classical seed-and-extend process. To achieve good mapping performances and to avoid over-correction of poorly mapped reads, we limit the amount of mismatches allowed according to a parameter ( 10 by default). Using the graph, the extend phase maps a read on several potential paths, and among all valid alignments, only those minimizing the number of mismatches are considered in a "best-first" approach similar to the BFC search. If several different optimal alignments exist, by default the read is not mapped. This choice can optionally be modified to output one of the optimal mappings. We highlight the fact that knowing the graph structure from the unitig set allows efficient graph exploration. Graph traversal based on a $k$-mer set would require to query the existence of all possible successors of every $k$-mer while our proposal needs to query the index only when the end of an unitig is reached. As an unitig can represent thousands of $k$-mers, this process is highly efficient and untractable in practice for $k$-mer spectrum correctors. In order to keep a low memory usage, Bgreat 2 uses a minimal perfect hash function [29] for indexing seeds. Moreover, it is possible to sub-sample seeds for indexation. For instance, by indexing only one out of ten seeds, we were able to run Bgreat2 on a human data set using around $20 \mathrm{~GB}$ of RAM at the price of only a slight decrease in correction efficiency (see Table 1). 
"output" — 2019/2/25 - 15:16 — page 5 — \#5

\begin{tabular}{|c|c|c|}
\hline Read: & AAATCGAGGCCCTCA & AAATCGAGGCCCTCA \\
\hline \multirow[t]{2}{*}{ De Bruijn graph: } & $\begin{array}{l}\text { CAAATCG CCCTGAC } \\
\text { ATCGAGGCCCT }\end{array}$ & CAAATCGAGGCCCTGAC \\
\hline & 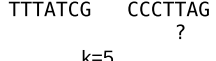 & TTTATCGAGGCCCTTAG \\
\hline
\end{tabular}

Fig. 5. Using a large $k$-mer size simplifies the graph by lowering the amount of unsolved repeats. In this example a repeat of size 10 is present in the genome in different contexts. With $k=5$, we are not able to correct the last nucleotide of the read represented by a blue arrow. But with $k=12$ we have determined the context of the repeat and know that only two possible paths exist. This way we are able to safely correct the read.

\subsection{4 $\boldsymbol{k}$-mer size selection}

We aim to use the highest possible $k$ value. This has the effect to resolve repeats smaller than $k$, thereby improving the correction as shown on Figure 5. However, choosing a $k$ value too large would yield a fragmented graph. Therefore, we implemented an automated tool, somewhat similar to $k$-merGenie [30], that uses ntCard to estimate the $k$-mer spectrum of the data set for several values of $k$. Our approach detects the first local minimum for each $k$-mer spectrum then selects the highest $k$ value for which this minimum is above the unitig threshold. This way, we expect to keep most genomic $k$-mers that are more abundant than the unitig threshold. This approach is more conservative and simpler than the one implemented in $k$-merGenie, which attempts to fit the $k$-mer spectrum on a haploid or diploid model with the aim of finding the $k$ value most suitable for assembling the reads.

\section{Results}

We present results based on simulated data sets as well as on real ones. Simulations make it possible to precisely evaluate correction metrics (Section 3.2) and to assess their impact on downstream assembly (Section 3.3). Correction evaluation was performed using simulated reads from several reference genomes: C. elegans, the human chromosome 1 , and the whole human genome. By contrast, the results presented in Section 3.4 aim to validate our approach using real data. All experiments were performed on a cluster node with a Xeon E5 2.8 GHz 24-core CPU, $256 \mathrm{~GB}$ of memory, and a mechanical hard drive. Our results are compared with those obtained using several state-of-the-art short-read correctors: Bloocoo [14], Musket [13], BFC [16], and Lighter [15]. We tried to include LoRDEC and Rcorrector in our benchmark (since those correctors rests on a principle similar to Bcool) but we finally removed them as we did not manage to obtain results on par with programs designed for correcting genomic short reads. In what follows, False Negatives $(F N)$ stand for noncorrected errors, whereas False Positives $(F P)$ are erroneous corrections and True Positives $(T P)$ are errors that were correctly corrected. The correction ratio is defined as $=\frac{T P+F N}{F N+F P}$; it is the ratio of the number of errors prior to correction $(T P+F N)$ vs. after correction $(F N+F P)$. The higher the correction ratio, the more efficient the tool.

\subsection{Performance benchmark}

Before presenting qualitative results, we first compare the performance of the correctors included in our benchmark. We evaluated the resources used by the different correctors on data sets simulated from the C. elegans and human genomes. We report here the memory used, the wall-clock time and the CPU time reported by the Unix time command. Our results, presented in Table 1, show that that Bcool has a higher RAM footprint and is slower than the other tools we tested, except Musket. This is due to Bcool's explicit graph representation and its indexation scheme. However, Bcool scales well with genome size, as shown in Table 1. Moreover, it is possible to reduce the memory footprint by sub-sampling during indexation the seeds

\begin{tabular}{|l|c|c|c|c|}
\hline Corrector & $\begin{array}{c}\text { RAM used } \\
(\mathrm{GB})\end{array}$ & $\begin{array}{c}\text { Wall-clock } \\
\text { time } \\
\text { (H:min) }\end{array}$ & $\begin{array}{c}\text { CPU } \\
\text { time } \\
\text { (H:min) }\end{array}$ & $\begin{array}{c}\text { Correction } \\
\text { ratio }\end{array}$ \\
\hline \multicolumn{5}{|c|}{ C. elegans } \\
\hline Bloocoo & 5.462 & $0: 07$ & $2: 02$ & 30.28 \\
Lighter & $\mathbf{0 . 6 2 7}$ & $\mathbf{0 : 0 6}$ & $\mathbf{1 : 4 0}$ & 31.16 \\
Musket & 24.755 & $0: 56$ & $16: 44$ & 90.33 \\
BFC & 8.390 & $0: 13$ & $4: 29$ & 14.58 \\
Bcool & 12.449 & $0: 21$ & $4: 25$ & $\mathbf{1 8 3 . 5 3}$ \\
\hline \multicolumn{5}{|c|}{ Human } \\
\hline Bloocoo & $\mathbf{1 0 . 5 0 0}$ & $9: 31$ & $90: 10$ & 6.79 \\
Lighter & 14.121 & $\mathbf{4 : 2 2}$ & $\mathbf{6 0 : 0 6}$ & 5.65 \\
Bcool & 48.081 & $11: 41$ & $129: 39$ & $\mathbf{2 3 . 8 4}$ \\
Bcool -i 5 & 19.724 & $15: 56$ & $194: 41$ & 22.621 \\
\hline
\end{tabular}

Table 1. Performance comparison on C. elegans and human simulated 250-bp reads with $1 \%$ error rate and $100 \mathrm{X}$ coverage using 20 cores. Bcool was run with a fixed $k$-mer size, $k=63$. Bcool -i 5 indexed only one out of every ten seeds to reduce memory usage. BFC and Musket were not able to correct the full human data sets.

used for read mapping. This results in a greatly reduced memory footprint at the price of a slight decrease in correction performance. In the human experiment, graph creation took $\approx 8 \mathrm{~h} 30$ and read mapping took $\approx 3 \mathrm{~h} 30$. As discussed below, there is clearly room for performance improvements both during the graph construction phase and the read mapping phase.

\subsection{Correction ratios on simulated data sets}

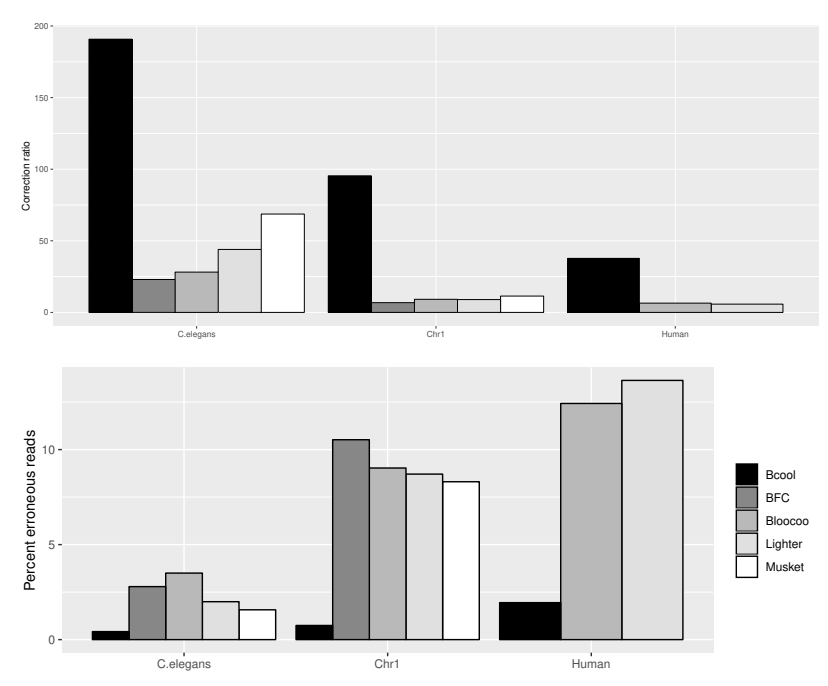

Fig. 6. Correction ratios (top) and percentage of erroneous reads after correction (bottom) for different correctors on our three simulated haploid data sets. We simulated 100x of $150 \mathrm{bp}$ reads with a $1 \%$ error rate. $\mathrm{BFC}$ and Musket ran out of memory on all full human data sets.

Our results on simulated haploid data are presented in Figure 6. They show that Bcool obtained a correction ratio an order of magnitude above the other tested tools. Note that, as shown in Supplementary materials we tested several other conditions (longer reads, lower coverage, lower error rate), all leading to the same conclusion. In each of our experiments, Bcool had a better correction ratio together with a better precision and recall. The correction precision is critical, as a corrector should not introduce 
new errors. Our experiments showed a good precision for all the tools we tested (even on a human genome), with a net advantage for Bcool though. For instance, our human-genome experiment with 100x coverage of $150 \mathrm{bp}$ reads yielded a precision of $95.33 \%$ for Bloocoo, $96.74 \%$ for Lighter and $99.61 \%$ for Bcool.

Diploid correction. To assess the impact of heterozygosity, we tested these correctors on simulated human diploid genomes. To obtain a realistic distribution of SNPs and genotypes, we used SNPs predicted from real human individuals and included them in the reference genome. Our results show that, on these data sets, the result quality of all tested correctors remains almost identical to those obtained on haploid simulations. The details and results of this experiment are presented in the Supplementary Material.

\subsection{Impact of the correction on assembly}

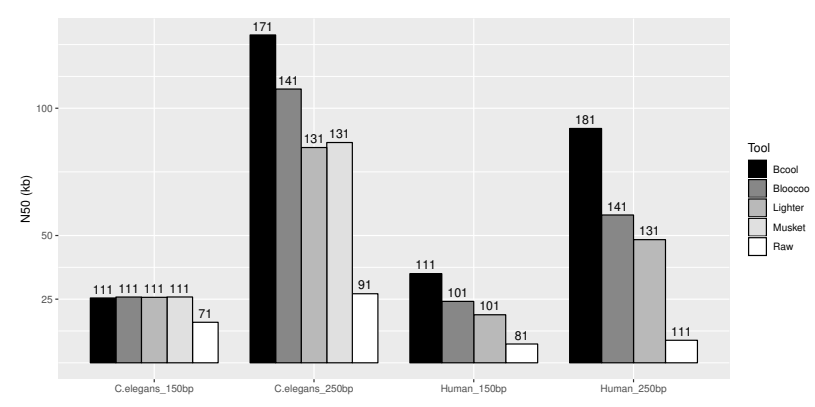

Fig. 7. Comparison of the N50s of the best assemblies obtained from the corrected simulated reads using Minia [31]. Only the best assembly obtained is kept and the corresponding $k$-mer size is indicated above each bar.

In order to evaluate the impact of the correction on assembly, we ran the Minia [31] assembler on uncorrected reads and on read sets corrected with each of the correctors included in our benchmark. For each assembly, we tested several $k$-values (the main parameter of Minia), from $k=21$ to $k=141$ with a step of 10 . For each corrector, only the best result is presented here. These results, presented in Figure 7, show that the N50 assembly metric is better on data corrected by Bcool. This can be explained by the fact that with a better read correction, a higher $k$ value can be selected, leading to a more contiguous assembly. As an example, for the $C$. elegans genome with 250-bp reads the best $k$-mer size was 91 for the raw reads, 131 for reads corrected using Lighter or Musket, 141 for reads corrected using Bloocoo and 171 for reads corrected using Bcool.

\subsection{Real data sets}

In this section we evaluate the impact of the correction on assembly continuity using several real data sets: a C. elegans Illumina HiSeq 2500 run with 79.8 millions reads of length $150 \mathrm{bp}$ amounting to $12 \mathrm{Gbp}$ (DRR050374) $(\approx 120 X$ coverage); and a A. thaliana Illumina MiSeq run with 33.6 millions reads of length $250 \mathrm{bp}$ amounting to $8.4 \mathrm{Gbp}$ $($ ERR2173372) $(\approx 60 X$ coverage). For this benchmark we used the string graph assembler fermi [32] given its efficiency and robustness. Assembly reports provided by Quast [33] are presented in Tables 2 and 3 using contigs larger than 1000 nucleotides. We see that on both datasets that Bcool obtains the most contiguous assembly in view of both N50 and N75 statistics.

\begin{tabular}{|l|c|c|c|c|c|}
\hline Corrector & N50 & NGA50 & N75 & NGA75 & Nb contigs \\
\hline Bcool & $\mathbf{2 3 , 0 2 1}$ & $\mathbf{2 0 , 6 9 6}$ & $\mathbf{1 0 , 8 8 0}$ & $\mathbf{8 , 5 5 2}$ & 8,373 \\
BFC & 19,524 & 17,872 & 9,490 & 7,642 & 9,235 \\
Bloocoo & 20,968 & 18,960 & 10,105 & 7,993 & 8,836 \\
Lighter & 21,435 & 19,269 & 10,254 & 8,122 & 8,714 \\
Musket & 22,545 & 20,291 & 10,788 & 8,545 & $\mathbf{8 , 3 6 8}$ \\
\hline
\end{tabular}

Table 2. Results of assembling the Illumina dataset DRR05374 (C. elegans) using fermi following various read correctors.

\begin{tabular}{|l|c|c|c|c|c|}
\hline Corrector & N50 & NGA50 & N75 & NGA75 & Nb contigs \\
\hline Bcool & $\mathbf{3 4 , 2 0 6}$ & $\mathbf{2 4 , 4 6 6}$ & $\mathbf{1 6 , 6 9 0}$ & $\mathbf{9 , 3 0 1}$ & 8,419 \\
BFC & 29,002 & 21,634 & 15,363 & 9,254 & $\mathbf{8 , 3 7 6}$ \\
Bloocoo & 27,737 & 20,751 & 14,281 & 8,783 & 8,783 \\
Lighter & 25,274 & 19,325 & 13,194 & 8,277 & 9,420 \\
Musket & 27,348 & 20,453 & 14,021 & 8,628 & 9,139 \\
\hline
\end{tabular}

Table 3. Results of assembling the Illumina dataset ERR2173372 (A. thaliana) using fermi following various read correctors.

\section{Perspective}

\subsection{Perspectives regarding short reads}

We have shown how to construct and clean a reference graph that can be used to efficiently correct sequencing errors. This approach is not to be compared with multiple- $k$ assembly as here we only apply a conservative correction to the graph without trying to remove variants nor to apply heuristics to improve the graph continuity: only $k$-mers that are very likely to be erroneous are removed in this process. Such conservative modifications on an intermediate graph used as a reference appears to us a promising approach to better exploit the high accuracy of short reads. The use of a high $k$-mer size is critical to address the correction problem on large, repeat-rich genomes, and the impossibility of $k$-merspectrum correctors to use a large $k$-mer size is a major limitations of such approaches. In contrast, our DBG-based solution uses a large $k$-mer size and therefore yields a more efficient correction on large, repeat-rich genomes. The resulting error-corrected reads are nearly perfect and can be assembled using an overlap-graph algorithm or may be used for other applications, such as variant calling.

Several propositions can be made to further improve Bcool. The read mapping step could make use of the quality values available in FASTQ files or provide other types of correction, such as read trimming. Adding the capacity to detect and correct indels during the mapping step could allow Bcool to correct other types of sequencing data, such as Ion torrent or PacBio CCS reads. The performance of the pipeline could also be globally improved. The de Bruijn graph construction could implement techniques similar to the sub-sampling used by Lighter in order to reduce its reliance on disk and therefore improve its running time. Besides, our mapping method is still naive, and implementing efficient heuristics such as the ones used by BWA [34] and Bowtie2 [35] could greatly improve the throughput of Bcool without decreasing the quality of the alignment. The mapping step could also take into account the pairing information to provide more precise alignments. Our $k$-mer-spectrum analysis could also be improved to choose a more accurate $k$-mer size and abundance filter at both $k$ mer and unitig level on real, on haploid or heterozygous data. From a more theoretical viewpoint, a study of whether using successively multiple $k$-mer sizes provides an even better correction (albeit at the price of a longer running time) would be an interesting perspective. Last but not least, another possible development could look into applications to data sets with highly heterogeneous coverage, as observed in single-cell, transcriptome or metagenome sequencing data. 
"output" — 2019/2/25 - 15:16 — page 7 — \#7

\subsection{Perspectives regarding long reads}

Surprisingly, the idea of aligning reads on a de Bruijn graph was applied to long, noisy reads before short, accurate ones. The efficiency of LoRDEC [20] and Bcool, respectively on long and short reads, suggests that such DBG-based correction is a general approach that can be applied to various kind of data sets. Short reads can also be used in conjunction with long reads, as for correcting systematic errors such as ONT homopolymers [9].

Using nearly perfect short reads as those corrected by Bcool may also improve long read correction. To test it, we simulated both short and long reads from the C.elegans reference genome and compared the amount of errors still present in the long reads after LoRDEC hybrid correction by mapping them on the reference with BWA. A coverage of $100 \mathrm{X}$ of short reads of $150 \mathrm{bp}$ were simulated along with long reads with $12 \%$ error rate using Pbsim [36]. Applying LoRDEC using non-corrected short reads lead to a $3.04 \%$ error rate on corrected long reads. When the short reads were first corrected using Bcool, the error rate on the corrected long reads fell to $2.33 \%$ (a $30.5 \%$ improvement). Additional work will be required to explore this idea further.

Last but not least, in the current context of decreasing error rates for long reads, we may soon reach a point at which $k$-mer or DBGbased technique will manage to perform efficient de novo reference-based correction using long reads alone. LORMA [21] is a first such attempt at using de Bruijn graph created from long reads to perform pure correction in an iterative manner. This suggests that de Bruijn graphs still have a bright future in bioinformatics.

\section{Acknowledgment}

We thank Romain Feron and Camille Marchet for their support and implication in this project. Antoine Limasset's postdoctoral position is funded by the Fédération Wallonie-Bruxelles via a "Action de Recherche Concertée" (ARC) grant to Jean-François Flot. Computational resources were provided by the Consortium des Équipements de Calcul Intensif (CÉCI), funded by the Fonds de la Recherche Scientifique de Belgique (F.R.S.-FNRS) under Grant No. 2.5020.11, as well as by GenOuest platform (genouest.org).

\section{References}

[1]Ruiqiang Li, Yingrui Li, Xiaodong Fang, Huanming Yang, Jian Wang, Karsten Kristiansen, and Jun Wang. SNP detection for massively parallel whole-genome resequencing. Genome Research, 19(6):11241132, 2009.

[2]Mark A DePristo, Eric Banks, Ryan Poplin, Kiran V Garimella, Jared R Maguire, Christopher Hartl, Anthony A Philippakis, Guillermo Del Angel, Manuel A Rivas, Matt Hanna, et al. A framework for variation discovery and genotyping using nextgeneration dna sequencing data. Nature Genetics, 43(5):491-498, 2011.

[3]Niranjan Nagarajan and Mihai Pop. Parametric complexity of sequence assembly: theory and applications to next generation sequencing. Journal of Computational Biology, 16(7):897-908, 2009.

[4]Job Dekker, Karsten Rippe, Martijn Dekker, and Nancy Kleckner. Capturing chromosome conformation. Science, 295(5558):13061311, 2002.

[5]Jacob O Kitzman. Haplotypes drop by drop: short-read sequencing provides haplotype information when long DNA fragments are barcoded in microfluidic droplets. Nature Biotechnology, 34(3):296299, 2016.
[6]Sarah Yeo, Lauren Coombe, Justin Chu, René L Warren, and Inanç Birol. ARCS: scaffolding genome drafts with linked reads. Bioinformatics, 2017.

[7]Hervé Marie-Nelly, Martial Marbouty, Axel Cournac, Jean-François Flot, Gianni Liti, Dante Poggi Parodi, Sylvie Syan, Nancy Guillén, Antoine Margeot, Christophe Zimmer, et al. High-quality genome (re)assembly using chromosomal contact data. Nature Communications, 5, 2014.

[8]Jean-François Flot, Hervé Marie-Nelly, and Romain Koszul. Contact genomics: scaffolding and phasing (meta)genomes using chromosome 3D physical signatures. FEBS Letters, 589(20):2966-2974, 2015.

[9]Miten Jain, Hugh E Olsen, Benedict Paten, and Mark Akeson. The Oxford Nanopore MinION: delivery of nanopore sequencing to the genomics community. Genome Biology, 17(1):239, 2016.

[10]Ryan R Wick, Louise M Judd, Claire L Gorrie, and Kathryn E Holt. Unicycler: resolving bacterial genome assemblies from short and long sequencing reads. PLOS Computational Biology, 13(6):e1005595, 2017.

[11]Pavel A Pevzner, Haixu Tang, and Michael S Waterman. An Eulerian path approach to DNA fragment assembly. Proceedings of the National Academy of Sciences, 98(17):9748-9753, 2001.

[12]Isaac Akogwu, Nan Wang, Chaoyang Zhang, and Ping Gong. A comparative study of $k$-spectrum-based error correction methods for next-generation sequencing data analysis. Human Genomics, 10(2):20, 2016

[13] Yongchao Liu, Jan Schröder, and Bertil Schmidt. Musket: a multistage $k$-mer spectrum-based error corrector for Illumina sequence data. Bioinformatics, 29(3):308-315, 2012.

[14]Gaëtan Benoit, Dominique Lavenier, Claire Lemaitre, and Guillaume Rizk. Bloocoo, a memory efficient read corrector. In European Conference on Computational Biology (ECCB), 2014.

[15]Li Song, Liliana Florea, and Ben Langmead. Lighter: fast and memory-efficient sequencing error correction without counting Genome Biology, 15(11):509, 2014.

[16]Heng Li. BFC: correcting Illumina sequencing errors. Bioinformatics, 31(17):2885-2887, 2015.

[17]Jan Schröder, Heiko Schröder, Simon J Puglisi, Ranjan Sinha, and Bertil Schmidt. SHREC: a short-read error correction method. Bioinformatics, 25(17):2157-2163, 2009.

[18]Leena Salmela and Jan Schröder. Correcting errors in short reads by multiple alignments. Bioinformatics, 27(11):1455-1461, 2011.

[19]Xiao Yang, Sriram P Chockalingam, and Srinivas Aluru. A survey of error-correction methods for next-generation sequencing. Briefings in Bioinformatics, 14(1):56-66, 2012.

[20]Leena Salmela and Eric Rivals. LoRDEC: accurate and efficient long read error correction. Bioinformatics, 30(24):3506-3514, 2014.

[21]Leena Salmela, Riku Walve, Eric Rivals, and Esko Ukkonen. Accurate self-correction of errors in long reads using de Bruijn graphs. Bioinformatics, 33(6):799-806, 2016.

[22]Li Song and Liliana Florea. Rcorrector: efficient and accurate error correction for Illumina RNA-seq reads. GigaScience, 4(1):48, 2015.

[23]Rayan Chikhi, Antoine Limasset, Shaun Jackman, Jared T Simpson, and Paul Medvedev. On the representation of de Bruijn graphs. In RECOMB, volume 8394, pages 35-55. Springer, 2014.

[24]Hamid Mohamadi, Hamza Khan, and Inanc Birol. ntCard: a streaming algorithm for cardinality estimation in genomics data. Bioinformatics, 33(9):1324-1330, 2017.

[25]Rayan Chikhi, Antoine Limasset, and Paul Medvedev. Compacting de Bruijn graphs from sequencing data quickly and in low memory. Bioinformatics, 32(12):i201-i208, 2016.

[26]BTRIM de Bruijn graph TRIMming tool. https://github. com/Malfoy/BTRIM. 
[27]BGREAT2 improved version of BGREAT. https://github. $\mathrm{com} / \mathrm{Mal}$ foy/BGREAT2.

[28]Antoine Limasset, Bastien Cazaux, Eric Rivals, and Pierre Peterlongo. Read mapping on de Bruijn graphs. BMC Bioinformatics, 17(1):237, 2016.

[29]Antoine Limasset, Guillaume Rizk, Rayan Chikhi, and Pierre Peterlongo. Fast and scalable minimal perfect hashing for massive key sets. arXiv preprint arXiv:1702.03154, 2017.

[30]Rayan Chikhi and Paul Medvedev. Informed and automated $k$-mer size selection for genome assembly. Bioinformatics, 30(1):31-37, 2013.

[31]Rayan Chikhi and Guillaume Rizk. Space-efficient and exact de Bruijn graph representation based on a Bloom filter. Algorithms for Molecular Biology, 8(1):22, 2013.

[32]Heng Li. Exploring single-sample SNP and INDEL calling with whole-genome de novo assembly. Bioinformatics, 28(14):1838-1844,
2012.

[33]Alexey Gurevich, Vladislav Saveliev, Nikolay Vyahhi, and Glenn Tesler. Quast: quality assessment tool for genome assemblies. Bioinformatics, 29(8):1072-1075, 2013.

[34]Heng Li and Richard Durbin. Fast and accurate short read alignment with Burrows-Wheeler transform. Bioinformatics, 25(14):17541760, 2009.

[35]Ben Langmead and Steven L Salzberg. Fast gapped-read alignment with Bowtie 2. Nature Methods, 9(4):357-359, 2012.

[36]Yukiteru Ono, Kiyoshi Asai, and Michiaki Hamada. PBSIM: PacBio reads simulator - toward accurate genome assembly. Bioinformatics, 29(1):119-121, 2012. 